\title{
More on the connection between planar field theory and string theory ${ }^{1}$
}

\author{
Korkut Bardakci \\ Department of Physics, University of California at Berkeley, \\ Berkeley, California 94720, U.S.A. \\ Theoretical Physics Group, Lawrence Berkeley National Laboratory, \\ University of California, Berkeley, California 94720, U.S.A. \\ E-mail: kbardakci@lbl.gov
}

ABSTRACT: We continue work on the connection between world sheet representation of the planar $\phi^{3}$ field theory and string formation. The present article, like the earlier work, is based on the existence of a solitonic solution on the world sheet, and on the zero mode fluctuations around this solution. The main advance made in this paper is the removal of the cutoff and the transition to the continuum limit on the world sheet. The result is an action for the modes whose energies remain finite in this limit (light modes). The expansion of this action about a dense background of graphs on the world sheet leads to the formation of a string.

KEYWords: Bosonic Strings, 1/N Expansion

ARXiv EPRINT: 0912.1304

\footnotetext{
${ }^{1}$ This manuscript has been authored by Korkut Bardakci under Contract No. DE-AC02-05CH11231 with the U.S. Department of Energy. The United States Goverment retains and the publisher, by accepting the article for publication, acknowledges that the United States Goverment retains a non-exclusive, paidup, irrevocable, world-wide license to publish or reproduce the published form of this manuscript, or allow others to do so, for United States Goverment purposes.
} 


\section{Contents}

1 Introduction $\quad 1$

2 The world sheet picture $\quad 4$

3 The world sheet field theory 5

4 Bosonization $\quad 8$

5 The classical solution $\quad 10$

6 The continuum limit 13

$\begin{array}{lll}7 & \text { The light sector of the model } & 15\end{array}$

8 The light sector action and string formation $\quad 18$

$\begin{array}{lll}9 & \text { Four transverse dimensions } & 19\end{array}$

10 Conclusions $\quad 22$

\section{Introduction}

This article is the continuation of the previous work on the same subject [1]. The basic problem, investigated in a series of papers [1-3], was to develop a string formulation for the planar graphs of the $\phi^{3}$ field theory on the world sheet. Both the present work and references $[1,2]$ are based on the world sheet picture developed in [3], which was in turn inspired by 't Hooft's seminal work [4]. Our motivation for writing a paper which has quite a bit of overlap with [1] is to clarify and extend the results obtained there, as well as to make a correction. To make the material intelligible to a reader not familiar with the previous work, we have inevitably to do some reviewing. We will start by briefly reviewing the main results of [1], and then summarize the advances made in this paper in comparison to [1]. As we go along, we will also preview each section, and again stressing the overlap and the differences with the previous work.

The starting point of reference [1] was the world sheet field theory developed in [2]. In section 2, we review the world sheet description of the planar graphs of the $\phi^{3}$ theory [3, 4], and in section 3, the world sheet field theory that reproduces these graphs [2] is described. The theory is formulated in terms a complex scalar $\phi$ and a two component fermion $\psi_{1,2}$; a central role is played by the world sheet field $\rho$, which is a composite of fermions (eq. (3.5)). Roughly speaking, $\rho$ measures the density of graphs on the world sheet. An important question is whether $\rho_{0}$, the average or the expectation value of $\rho$, is different from zero. 
$\rho_{0}$ vanishes in any finite order of perturbation theory, whereas a non-zero $\rho_{0}$ means that the world sheet is densely covered by graphs, and the contribution of higher order graphs dominate. In references [1] and [2], it was argued, on the basis of mean field approximation, that the ground state of the model was realized in the $\rho_{0} \neq 0$ phase. We would like to stress that $\rho_{0}=0$ and $\rho_{0} \neq 0$ are two different phases of the model, analytically disconnected from each other. Here $\rho_{0}$ plays the role of an order parameter which distinguishes between phases, similar to, for example, what happens in the Higgs effect.

In this paper, we will not investigate the ground state of this unphysical model, which may not even exist. Instead, we will have a more modest goal: Do the graphs that are dense on the world sheet have a string description? It seems plausible that such set of graphs would lead to a Nambu-Goto type action and in fact, this idea motivated some of the early work on this subject $[5,6]$. To answer this question, we will simply fix $\rho_{0}$ at a non-vanishing value by introducing a source coupled to $\rho$ and suitably tuning this source. This is equivalent to choosing by hand a set of graphs whose average density is $\rho_{0}$ and investigating their properties. Here we do not probe too deeply into the dynamics of the $\phi^{3}$ theory; on the other hand, the results obtained are more robust, and hopefully, they are more generally applicable, possibly to more physical models, like gauge theories. One would then like to compute the corresponding effective potential by the mean field method or any other reasonable method, and determine the ground state by finding its minimum as a function of $\rho_{0}$. Hopefully, the ground state will have $\rho_{0} \neq 0$, leading to string formation.

In section 4, which is a partial review of a corresponding section in [1], the world sheet fermions are bosonized. In the fermionic picture, $\rho$ takes on two values, 0 and 1 , corresponding to the presence or the absence of a line on the world sheet. In contrast, in the bosonic picture, $\rho$ is a continuous field with values ranging from 0 to 1 . The bosonic picture provides a very convenient setup for the mean field approximation, developed in the following sections. This section also includes a correction to [1]: In that reference, it was incorrectly stated that $\rho$ is a non-dynamical time independent field. Here we show that, on the contrary, $\rho$ is a time dependent dynamical field, and it plays an important role in the dynamics of the string.

A crucial feature of the world sheet field theory is that it has solitonic classical solutions. In fact, the trivial vacuum of the theory is unstable against tadpole emissions, and the model is stablized by introducing the solitonic background. These classical solutions were studied in [1] and [3], and in section 5, we briefly review them. An unusual feature is that the corresponding classical energy is ultraviolet divergent. The degree of divergence depends on number of dimensions; in most of the paper, we will take the number of transverse dimensions $\mathrm{D}$ to be two (4 space-time dimensions). This simplifies the treatment considerably: The model is superrenormalizable, the classical energy is log divergent, and the coupling constant is finite. This divergence can be removed by mass renormalization. In [1], this renormalization was carried out for a specific value of $\rho_{0}$ corresponding to a candidate ground state; here, since $\rho_{0}$ is variable at our disposal, we have to require renormalizibility for all $\rho_{0}$ in the physical range $0 \leq \rho_{0} \leq 1$. This is a much stronger condition, and it fixes part of the world sheet action.

To see how this comes about, consider the interaction vertex where three propagators 
meet (figure 3 ). The prefactor $1 /\left(2 p^{+}\right)$in the propagator (eq. (2.1)) has to be attached to the vertex, and there are various ways of doing this. In [1], these prefactors were attached to the vertex symmetrically, as in eq. (3.9), but there are also asymmetrical ways of doing it. So long as $\rho$ is a discrete variable with values 0 and 1 , as in the original fermionic version, all of these vertices yield the same result, but once $\rho$ becomes a continuous variable, this is no longer true. In section 5, we show that requiring renormalizibility for arbitrary $\rho$ fixes the vertex completely (eq. (5.6)). In contrast to the symmetrical vertex used in [1], this corresponds to an asymmetrical vertex. Apart from this issue of renormalization, its precise form will not matter for any of the other results of this article.

As explained in sections 2 and 3, to have a well defined field theory, one of the coordinates of the world sheet, $\sigma$, is discretized in steps of "a". This amounts to compactifying the light cone coordinate $x^{-}$, and it was extensively used both in field theory [7], and in string theory [8] and also M theory [9]. In section 6, we study in some detail the continuum limit $a \rightarrow 0$ on the world sheet. This limit was not investigated systematically in [1], and it is an important problem, since the world sheet field theory greatly simplifies and a string picture clearly emerges only in this limit. An undesirable feature of the field theory with discretized $\sigma$ was its non-locality in this coordinate. In the limit $a \rightarrow 0$, the non-locality goes away, and the result is a local field theory on the world sheet, with an action with no higher than first order $\sigma$ derivative of the fields. The derivation of a local world sheet theory in the continuum limit is one of central results of the present work that goes beyond reference [1].

The continuum hamiltonian can be written as a sum of two terms: One that is finite in the $a \rightarrow 0$ limit and the other that blows up as $1 / a^{2}$ (eq. (6.8)). In section 7 , we address the question of how to interpret this result. At first sight, it may appear that the energies and therefore the masses of various modes go to infinity. There are, however two exceptions to this observation. With some natural scaling of parameters, the mass of the $\rho$ stays finite, and there is also a transverse vector mode $\mathbf{v}$, whose mass is vanishes. This is easy to understand: The model is invariant under the translation of the momentum $\mathbf{q}$ (eqs. $(2.2)$ and (3.13)), but this invariance is spontanously broken by the presence of the soliton, and $\mathbf{v}$ is the corresponding Goldstone mode. This mode was discovered in [1] by expanding to quadratic order about the classical background; here, using translation invariance, we derive an exact result, valid to all orders.

We call the two modes discussed above the light modes, and we assume all the other modes, whose masses go to infinity in the continuum limit, decouple from the light modes. This hypothesis of the decoupling of heavy modes is widely used both in field theory and string theory. In section 8 , using this hypothesis, we derive the continuum limit, resulting in a world sheet action in terms of the light modes $\rho$ and $\mathbf{v}$ alone (eq. (8.2)), which is the main result of this work. All reference to the cutoff "a" and the momentum q has disappeared from the problem. Expanding about a constant background $\rho=\rho_{0}$ results in a free massive $\rho$ and a free string action for $\mathbf{v}$.

In section 9 , we extend these results to $D=4(5+1$ dimensions). The main complication is a cutoff dependent coupling constant, characteristic of $\phi^{3}$ in 6 dimensions, which is asymptotically free. Despite this complication, everything works out pretty much the same 


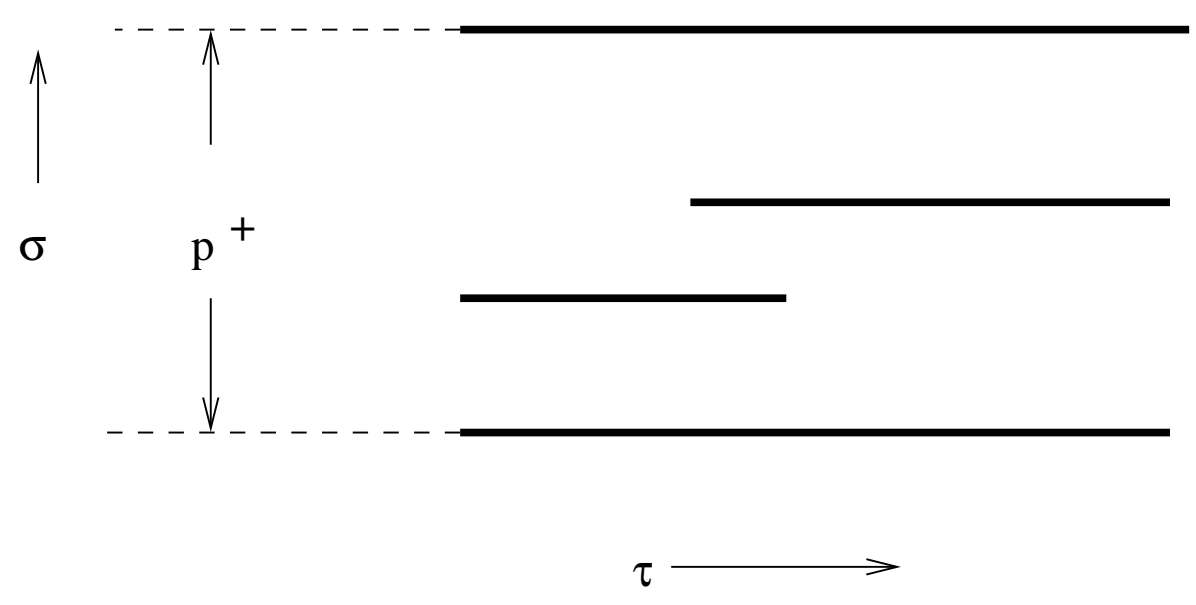

Figure 1. A typical graph.

as in $D=2$, and a string is formed from the light mode $\mathbf{v}$. The main difference is that the dimensionless coupling constant is replaced by a mass parameter, and the theory can be renormalized by identifying this mass with the slope of the string. What happens here is similar to what happens in field theory when one expands around a non-trivial background such as a soliton or an instanton: Although the background is non-perturbative, the short distance behaviour and properties like asymptotic freedom are the same as in perturbation theory. Finally, section 10 summarizes our conclusions.

\section{The world sheet picture}

The planar graphs of $\phi^{3}$ in the light cone representation of 't Hooft [4] have a simple represantation. The world sheet is parametrized by the coordinates

$$
\tau=x^{+}=\left(x^{0}+x^{1}\right) / \sqrt{2}, \quad \sigma=p^{+}=\left(p^{0}+p^{1}\right) / \sqrt{2} .
$$

The coordinate $\tau$ will serve as the light cone time. A general plane graph is represented by a collection of horizontal solid lines (figure 1), where the n'th line carries a D dimensional transverse momentum $\mathbf{q}_{\mathbf{n}}$. Two adjacent solid lines labeled by $\mathrm{n}$ and $\mathrm{n}+1$ correspond to the light cone propagator

$$
\Delta\left(\mathbf{p}_{\mathbf{n}}\right)=\frac{\theta(\tau)}{2 p^{+}} \exp \left(-i \tau \frac{\mathbf{p}_{\mathbf{n}}^{2}+m^{2}}{2 p^{+}}\right)
$$

where $\mathbf{p}_{\mathbf{n}}=\mathbf{q}_{\mathbf{n}}-\mathbf{q}_{\mathbf{n}+\mathbf{1}}$ is the momentum flowing through the propagator. The interaction takes place at the beginning and at the end of each line, where a factor of $g$, the coupling constant is inserted. Ultimately, one has to integrate over all possible locations and lengths of solid lines, as well as over the momenta they carry.

We note that there is invariance under the translation

$$
\mathbf{q}_{\mathbf{n}} \rightarrow \mathbf{q}_{\mathbf{n}}+\mathbf{r},
$$




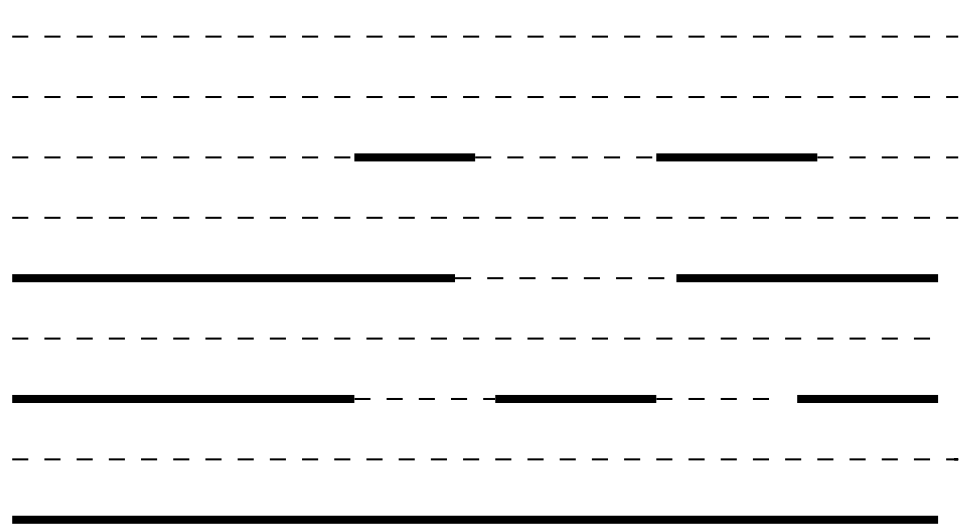

Figure 2. Solid and dotted lines.

where $\mathbf{r}$ is a constant vector. This invariance will play an important role in the subsequent development.

To avoid singular expressions later on, it is convenient to discretize the coordinate $\sigma$ in steps of length a, which amounts to compactifying the light cone coordinate $x^{-}=$ $\left(x^{0}-x^{1}\right) / \sqrt{2}$ at radius $R=1 / a$. This type of compactification has proved useful in field theory [7] and also in string theory [8], and in the $\mathrm{M}$ theory [9]. In contrast, the time coordinate $\tau$ will remain continuous. A useful way of visualizing the discretized world sheet is pictured in figure 2. The boundaries of the propagators are marked by solid lines as before; but now, the bulk is filled by dotted lines spaced a distance a apart.

We have also to specify the boundary conditions on the world sheet. The $\sigma$ coordinate is compactified by imposing periodic boundary conditions at $\sigma=0$ and $\sigma=p^{+}$, where $p^{+}$ is the total + component of the momentum flowing the whole graph. This means that the total transverse momentum $\mathbf{p}$ flowing through the graph is zero. In contrast, the boundary conditions at $\tau= \pm \infty$ will be left free.

\section{The world sheet field theory}

In this section, we will briefly review the world sheet theory developed in [1], which reproduces the light cone graphs described in the previous section. We introduce the complex scalar field $\phi(\sigma, \tau, \mathbf{q})$ and its conjugate $\phi^{\dagger}$, which at time $\tau$ annihilate and create a solid line carrying momentum $\mathbf{q}$ and located at site labeled by $\sigma$. They satisfy the usual commutation relations

$$
\left[\phi(\sigma, \tau, \mathbf{q}), \phi^{\dagger}\left(\sigma^{\prime}, \tau, \mathbf{q}^{\prime}\right)\right]=\delta_{\sigma, \sigma^{\prime}} \delta\left(\mathbf{q}-\mathbf{q}^{\prime}\right) .
$$

The vacuum, defined by

$$
\phi(\sigma, \tau, \mathbf{q})|0\rangle=0,
$$

corresponds to a state with only dotted lines (empty world sheet). By applying $\phi^{\dagger}$ 's on the vacuum, one can construct states with arbitrary number of solid lines. 
In addition, we will need a two component fermion field $\psi_{i}(\sigma, \tau), i=1,2$, and its adjoint $\bar{\psi}_{i}$, with the standard anticommutation relations

$$
\left[\psi_{i}(\sigma, \tau), \bar{\psi}_{i^{\prime}}\left(\sigma^{\prime}, \tau\right)\right]_{+}=\delta_{i, i^{\prime}} \delta_{\sigma, \sigma^{\prime}}
$$

and propagate freely on an uniterrupted line. The fermion with $i=1$ lives on the dotted lines and the one with $i=2$ lives on the solid lines. The main reason for introducing the fermions is to avoid unwanted configurations on the world sheet. One type of such a configuration corresponds to multiple solid lines at the same site, generated by repeated applications of $\phi^{\dagger}$ at the same $\sigma$. To get rid of these redundant configurations, we impose the constraint

$$
\int d \mathbf{q} \phi^{\dagger}(\sigma, \mathbf{q}) \phi(\sigma, \mathbf{q})=\rho(\sigma)
$$

where

$$
\rho=\bar{\psi}_{2} \psi_{2}
$$

which is equal to one on solid lines and zero on the dotted lines. This constraint ensures that there is at most one solid line at each site. Here and in the rest of the paper, it is understood that the fields $\phi, \rho$ as well as the fermionic fields are functions of both $\sigma$ and $\tau$, although for simplicity, we will frequently not write down explicitly the dependence on one or both of these variables.

Fermions are also needed to avoid another set of unwanted configurations: Propagators should be assigned only to adjacent solid lines; those associated with non-adjacent solid lines are not allowed. For this purpose, we define, for $\sigma_{j}>\sigma_{i}$

$$
\mathcal{E}\left(\sigma_{i}, \sigma_{j}\right)=\prod_{k=i+1}^{k=j-1}\left(1-\rho\left(\sigma_{k}\right)\right)
$$

If $\sigma_{j} \leq \sigma_{i}, \mathcal{E}$ is defined to be zero. The crucial property of this function is that it is equal to one only if the the two solid lines at $\sigma_{i}$ and $\sigma_{j}$ are seperated by dotted lines. If there are any solid lines in between, it is zero. With the help of $\mathcal{E}$, the free Hamiltonian (no interaction) can be written as

$$
\begin{aligned}
H_{0}= & \frac{1}{2} \sum_{\sigma, \sigma^{\prime}} \int d \mathbf{q} \int d \mathbf{q}^{\prime} \frac{\mathcal{E}\left(\sigma, \sigma^{\prime}\right)}{\sigma^{\prime}-\sigma}\left(\left(\mathbf{q}-\mathbf{q}^{\prime}\right)^{2}+m^{2}\right) \\
& \times \phi^{\dagger}(\sigma, \mathbf{q}) \phi(\sigma, \mathbf{q}) \phi^{\dagger}\left(\sigma^{\prime}, \mathbf{q}^{\prime}\right) \phi\left(\sigma^{\prime}, \mathbf{q}^{\prime}\right) \\
& +\sum_{\sigma} \lambda(\sigma)\left(\int d \mathbf{q} \phi^{\dagger}(\sigma, \mathbf{q}) \phi(\sigma, \mathbf{q})-\rho(\sigma)\right)
\end{aligned}
$$

where $\lambda$ is a lagrangian multiplier enforcing the constraint (3.4).

It is now not too difficult to verify that, the operator $\exp \left(-i \tau H_{0}\right)$, applied on the states described following eq. (3.2), reproduces a collection of free propagators given by (2.1), except for the prefactor $1 /\left(2 p^{+}\right)$. The constraint (3.4) eliminates unwanted states with multiple solid lines, and the presence of $\mathcal{E}$ ensures that propagators are generated only by adjacent solid lines. 


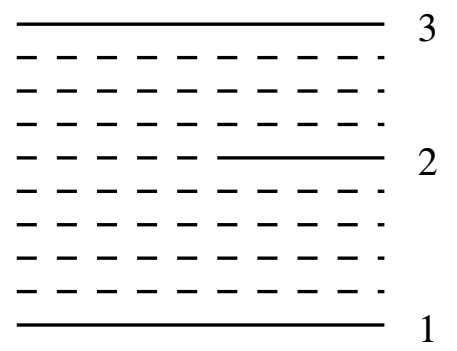

a)

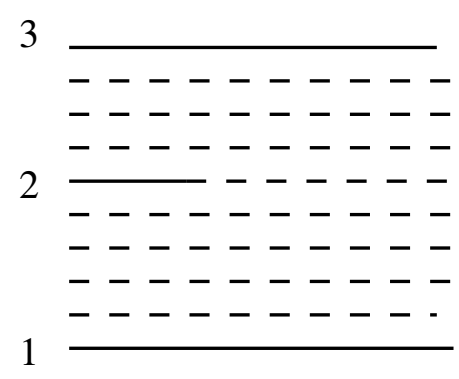

b)

Figure 3. The two $\phi^{3}$ vertices.

Taking advantage of (3.4), it is possible to rewrite the free hamiltonian in a simpler form:

$$
\begin{aligned}
H_{0}= & \frac{1}{2} \sum_{\sigma, \sigma^{\prime}} G\left(\sigma, \sigma^{\prime}\right)\left(\frac{1}{2} m^{2} \rho(\sigma) \rho\left(\sigma^{\prime}\right)+\rho\left(\sigma^{\prime}\right) \int d \mathbf{q} \mathbf{q}^{2} \phi^{\dagger}(\sigma, \mathbf{q}) \phi(\sigma, \mathbf{q})\right. \\
& \left.\quad-\int d \mathbf{q} \int d \mathbf{q}^{\prime}\left(\mathbf{q} \cdot \mathbf{q}^{\prime}\right) \phi^{\dagger}(\sigma, \mathbf{q}) \phi(\sigma, \mathbf{q}) \phi^{\dagger}\left(\sigma^{\prime}, \mathbf{q}^{\prime}\right) \phi\left(\sigma^{\prime}, \mathbf{q}^{\prime}\right)\right) \\
& +\sum_{\sigma} \lambda(\sigma)\left(\int d \mathbf{q} \phi^{\dagger}(\sigma, \mathbf{q}) \phi(\sigma, \mathbf{q})-\rho(\sigma)\right)
\end{aligned}
$$

where we have defined

$$
G\left(\sigma, \sigma^{\prime}\right)=\frac{\mathcal{E}\left(\sigma, \sigma^{\prime}\right)+\mathcal{E}\left(\sigma^{\prime}, \sigma\right)}{\left|\sigma-\sigma^{\prime}\right|} .
$$

It remains to introduce interaction. The two kinds of interaction vertices are pictured in figure 3. In the first one, the operator $\phi^{\dagger}$ creates the solid line 2, and in the second figure, $\phi$ destroys the solid line. This is, however, not the whole story; we have to take care of the missing factor of $1 /\left(2 p^{+}\right)$mentioned above by attaching it to the vertices. There many ways of doing this; in references [1,3], this was done by attaching a factor of

$$
V=\frac{1}{\sqrt{8 p_{12}^{+} p_{23}^{+} p_{13}^{+}}}=\frac{1}{\sqrt{8\left(\sigma_{2}-\sigma_{1}\right)\left(\sigma_{3}-\sigma_{2}\right)\left(\sigma_{3}-\sigma_{1}\right)}}
$$

to each vertex. This is equivalent to splitting the factor $1 /\left(2 p^{+}\right)$into two factors of $1 / \sqrt{2 p^{+}}$ and attaching one to the beginning and one to the end of the propagator. This is the most symmetric assignment, but one could consider other ways of splitting this factor, resulting in different expressions for $V$. Later on, we will choose to attach the whole factor to the beginning (or to the end) of each propagator. Of course, in the exact theory, all of these different assignments are equivalent; only when an approximation is made, the differences between them emerge.

Given a choice of $V$, we define

$$
W\left(\sigma_{2}\right)=\sum_{\sigma_{1}<\sigma_{2}} \sum_{\sigma_{2}<\sigma_{3}} \rho\left(\sigma_{1}\right) \mathcal{E}\left(\sigma_{1}, \sigma_{3}\right) \rho\left(\sigma_{3}\right) V\left(\sigma_{1}, \sigma_{2}, \sigma_{3}\right) .
$$


In this expression, the factor $\rho \mathcal{E} \rho$ multiplying $V$ picks the correct vertex configuration of figure 3 , and projects out the unwanted configurations. With the help of $W$, the interaction hamiltonian can be written as

$$
H_{I}=g \sum_{\sigma} \int d \mathbf{q} W(\sigma)\left(\phi(\sigma, \mathbf{q}) \rho_{+}(\sigma)+\phi^{\dagger}(\sigma, \mathbf{q}) \rho_{-}(\sigma)\right)
$$

where $g$ is the coupling constant, and $\rho_{ \pm}$are defined by

$$
\rho_{+}=\bar{\psi}_{1} \psi_{2}, \rho_{-}=\bar{\psi}_{2} \psi_{1}
$$

The additional factors of $\rho_{ \pm}$are needed to make sure that a solid line is always paired with an $i=2$ fermion and a dotted line with an $i=1$ fermion.

Finally, the total hamiltonian is given by

$$
H=H_{0}+H_{I}
$$

and the corresponding action by

$$
S=\int d \tau\left(\sum_{\sigma}\left(i \bar{\psi} \partial_{\tau} \psi+i \int d \mathbf{q} \phi^{\dagger} \partial_{\tau} \phi\right)-H(\tau)\right) .
$$

We note that the hamiltonian, as well as the action, is invariant under the transformation

$$
\phi(\sigma, \tau, \mathbf{q}) \rightarrow \phi(\sigma, \tau, \mathbf{q}+\mathbf{r})
$$

which is a restatement of (2.2) in the language of field theory. This invariance will play an important role later on.

\section{Bosonization}

The hamiltonian defined by eqs.(3.8) and (3.11) is so far exact. It is also non-local in the $\sigma$ direction and somewhat complicated. We will transform it into a local and simpler form later on. As a first step, in preparation for the mean field approximation, it is very convenient to bosonize the fermions defined in the previous section. This was done in [1], and we follow this reference closely. In addition to $\rho$ (eq. (3.5)), we introduce the bosonic field $\xi$ and set,

$$
\rho_{+}=\bar{\psi}_{1} \psi_{2}=\sqrt{\rho-\rho^{2}} e^{i \xi}, \rho_{-}=\bar{\psi}_{2} \psi_{1}=\sqrt{\rho-\rho^{2}} e^{-i \xi}
$$

The kinetic energy term for $\psi$ in eq. (3.12) is then replaced by

$$
\int d \tau \sum_{\sigma} i \bar{\psi} \partial_{\tau} \psi \rightarrow \int d \tau \sum_{\sigma} \xi \partial_{\tau} \rho
$$

One can check that that this action produces the correct equations of motion and the correct commutation relations for the fermionic bilinears. It is therefore an exact transformation. However, we note that bosonization has replaced discrete variables by continuous 
ones. For example, according to its original definition, $\rho$ could only take on the values 0 and 1 , but as an independent bosonic field, it can vary continuously between 0 and 1 . It natural to interpret it as the probability of finding a solid line at a given location. In going over to the bosonic picture, the problem has been reformulated in terms of a continuously valued field, but so long as the functional integral over $\rho$ is carried out exactly, the reformulation is exact. The approximation comes later: Unable to do the functional integral exactly, we will resort to the mean field approximation, which amounts to evaluating the integral approximately by expanding $\rho$ about a constant non-zero background $\rho_{0}$. Since, by its definition, $\rho_{0}$ measures the average density of solid lines on the world sheet, if $\rho_{0} \neq 0$, the world sheet is densely covered by solid lines, or equivalently, by Feynman graphs. As explained in the introduction, we will arrange to have $\rho_{0} \neq 0$ by adding an external field $J$ coupled to $\rho$ to the action (eq. (7.3)); the minimum of the potential for $\rho$ will then be at an adjustable $\rho=\rho_{0}$.

It is possible to eliminate the field $\xi$ from the problem, leaving behind only $\rho$. This is because the action is invariant under the transformation

$$
\begin{aligned}
\phi & \rightarrow \exp (-i \alpha) \phi, & \phi^{\dagger} & \rightarrow \exp (i \alpha) \phi^{\dagger}, \\
\xi & \rightarrow \xi+\alpha, & \lambda & \rightarrow \lambda-\partial_{\tau} \alpha,
\end{aligned}
$$

where $\alpha$ is an arbitrary function of $\sigma$ and $\tau$, so this is a gauge transformation on the world sheet. We can therefore fix the gauge by setting

$$
\xi=0
$$

The equation of motion with respect to $\xi$ at $\xi=0$ should then be imposed as a constraint:

$$
\partial_{\tau} \rho-i g \sqrt{\rho-\rho^{2}} W \int d \mathbf{q}\left(\phi-\phi^{\dagger}\right)=0
$$

Using the equations of motion, this can be shown to be equivalent to

$$
\partial_{\tau}\left(\rho-\int d \mathbf{q} \phi^{\dagger} \phi\right)=0
$$

This nothing but the time derivative of the constraint (3.4), so everything is consistent. In fact, this shows that (3.4) can be imposed as an initial condition, and it will then be preserved by the equations of motion. Eq. (4.4) replaces eq. (5.2) of reference [1], which was incorrect.

After bosonization, the interaction hamiltonian becomes

$$
H_{I} \rightarrow g \sum_{\sigma} W(\sigma) \int d \mathbf{q}\left(\phi(\sigma, \mathbf{q})+\phi^{\dagger}(\sigma, \mathbf{q})\right)
$$

where we have taken the liberty of absorbing the factor of $\sqrt{\rho-\rho^{2}}$ into the definition of $W$. 


\section{The classical solution}

In this section, we will treat $\rho(\sigma, \tau)$ and $\lambda(\sigma, \tau)$ as fixed classical external fields, with $\rho$ satisfying the condition

$$
0 \leq \rho \leq 1
$$

Of course, ultimately we have to integrate functionally over them, but for the time being, they will be fixed. The function $W$, which can be expressed in terms of $\rho$, also becomes an external field, and as a consequence, $H_{I}$, being linear in $\phi$ and $\phi^{\dagger}$, becomes a tadpole term, and destabilizes the vacuum of eq. (3.2). It is then natural to eliminate this term by shifting $\phi$ by

$$
\phi=\phi_{0}+\phi_{1}, \phi^{\dagger}=\phi_{0}^{\star}+\phi_{1}^{\dagger} .
$$

$\phi_{0}$ is then a classical field, determined by the tadpole elimination condition, and $\phi_{1}$ represents quantum fluctuations around this classical background. Although after this shift, $H_{I}$ disappears, interaction is now encoded in the expression for $\phi_{0}$, which depends on the coupling constant $g$ (see eq. (5.10)).

We choose $\phi_{0}$ so that it depends on the vector $\mathbf{q}$ only through $\mathbf{q}^{2}$ (rotation invariance). This ensures that the last term on the right of eq. (3.8) does not contribute, and we have the simple problem of a hamiltonian with only quadratic and linear terms. The tadpole elimination equation gives

$$
\phi_{0}=-g \frac{W}{E \mathbf{q}^{2}+\lambda}
$$

where,

$$
E(\sigma)=\frac{1}{2} \sum_{\sigma^{\prime}} G\left(\sigma, \sigma^{\prime}\right) \rho\left(\sigma^{\prime}\right)
$$

Although we have eliminated one set of tadpoles, a new term of the form

$$
\Delta S=\int d \tau \int d \mathbf{q} \sum_{\sigma}\left(i\left(\phi_{1}^{\dagger}-\phi_{1}\right) \partial_{\tau} \phi_{0}\right)
$$

is generated in the action. Being linear in $\phi_{1}$, this term is also a tadpole. If we choose $\rho$ to be $\tau$ independent, $\phi_{0}$ will also be $\tau$ independent, and this term will vanish. In this special case, $\phi_{0}$ is a classical solution to the equations of motion (a soliton), which was introduced in references [3] and [1]. For the time being, however, we will keep $\rho$ general and not suppress its $\tau$ dependence. We will later see that the resulting extra term is actually needed to provide $\rho$ with a kinetic energy term.

The classical contribution to the action is of special interest. In general, due to the integration over $\mathbf{q}$, it has an ultraviolet divergence, which depends on the dimension of the space-time. In what follows, we will specialize to $D=2$ (4 space-time dimensions), except in section 9 , when we will consider $D=4$ (6 space-time dimensions). At $D=2, \phi^{3}$ is super renormalizable, and only the mass term is logarithmically divergent. In our treatment, this 
divergence shows up in the classical contribution to the action:

$$
\begin{aligned}
S_{c} & =S\left(\phi=\phi_{0}\right)=g^{2} \int d \tau \sum_{\sigma}\left(\frac{\pi W^{2}}{E}+\pi \int_{0}^{\Lambda^{2}} d \mathbf{q}^{2} \frac{W^{2}}{E \mathbf{q}^{2}+\lambda}\right) \\
& =g^{2} \pi \int d \tau \sum_{\sigma} \frac{W^{2}}{E}\left(1+\ln \left(\frac{E \Lambda^{2}}{\lambda}\right)\right),
\end{aligned}
$$

where $\Lambda$ is an ultraviolet cutoff. In a Lorentz invariant theory, we expect this divergence to contribute only to the mass term in eq. (3.8), so it should be proportional to

$$
\begin{aligned}
S_{m} & =-\int d \tau \sum_{\sigma, \sigma^{\prime}}\left(\frac{1}{4} m^{2} G\left(\sigma, \sigma^{\prime}\right) \rho(\sigma) \rho\left(\sigma^{\prime}\right)\right) \\
& =-\frac{1}{2} m^{2} \int d \tau \sum_{\sigma} E(\sigma) \rho(\sigma) .
\end{aligned}
$$

Setting the cutoff term in $S_{c}$ proportional to $S_{m}$ gives

$$
\frac{W^{2}}{E}=\text { const. } E \rho \text {. }
$$

If this condition is satisfied, the divergence can be eliminated by a mass counter term. Setting the constant equal to one, which can always be arranged by a redefinition of the coupling constant $\mathrm{g}$, we have the defining equation

$$
W=E \sqrt{\rho}
$$

for the hitherto unspecified vertex function W. From now on, we will assume that the divergence has been eliminated by a counter term, so that $S_{c}$ can be dropped if we replace $\mathrm{m}$ by $m_{r}$, the renormalized mass.

We have derived this condition by requiring renormalizability for an arbitrary $\rho(\sigma, \tau)$. However, the same result can also be derived by attaching the factors $1 /\left(2 p^{+}\right)$(eq. (2.1)) always to the beginning (or always to the end) of each propagator. In contrast, a different distribution of this factor, such as the one in eq. (3.9), spoils renormalizability when the background $\rho$ is arbitrary. This is surprising, since in the exact theory, it should not matter how this factor is split between the beginning and the end of the propagator. This is true if $\rho$ is built out of fermions (eq. (3.5)) and therefore it is restricted to take on only the values one and zero. However, after bosonization, $\rho$ is a continuous variable, and different splittings of the factor $1 /\left(2 p^{+}\right)$give different results. We then choose the splitting that leads to eq. (5.6) and thereby makes mass renormalization possible. It should be stressed that apart from renormalizability, subsequent results we are going to obtain, concerning, for example string formation, are robust. They do not depend on the detailed form of $W$, but only on its general features.

So far we have treated $\lambda$ as a fixed external field, same as $\rho$. We are now ready to carry out the functional integration over $\lambda$. We first set

$$
\lambda=\lambda_{0}+\lambda_{1}
$$


where $\lambda_{0}$ is the classical part and $\lambda_{1}$ is the quantum fluctuation to be integrated over. It is now natural to redefine the classical solution $\phi_{0}$ (eq. (5.2)) by replacing $\lambda$ by its classical part $\lambda_{0}$ :

$$
\phi_{0} \rightarrow-g \sqrt{\rho} \frac{E}{E \mathbf{q}^{2}+\lambda_{0}} .
$$

Also, eq. (5.6) for $\mathrm{W}$ was used.

$\lambda_{0}$ is determined by the classical equation of motion it satisfies, or equivalently, by directly imposing the constraint (3.4) on the redefined $\phi_{0}$. The result is,

$$
\lambda_{0}=\pi g^{2} E
$$

and,

$$
\phi_{0}=-g \frac{\sqrt{\rho}}{\mathbf{q}^{2}+\pi g^{2}} .
$$

Making use of (5.7), (5.9) and (5.10), the term proportional to $\lambda$ in (3.7) can now be rewritten as

$$
\sum_{\sigma} \lambda\left(\int d \mathbf{q} \phi^{\dagger} \phi-\rho\right)=\sum_{\sigma}\left(\lambda_{0} \int d \mathbf{q} \phi_{1}^{\dagger} \phi_{1}+\lambda_{1} \int d \mathbf{q}\left(\phi_{0}\left(\phi_{1}+\phi_{1}^{\dagger}\right)+\phi_{1}^{\dagger} \phi_{1}\right)\right),
$$

and the integration over $\lambda_{1}$ gives the constraint

$$
\int d \mathbf{q}\left(\phi_{0}\left(\phi_{1}+\phi_{1}^{\dagger}\right)+\phi_{1}^{\dagger} \phi_{1}\right)=0
$$

Therefore, this term can be replaced by

$$
\sum_{\sigma} \lambda_{0} \int d \mathbf{q} \phi_{1}^{\dagger} \phi_{1}
$$

plus the constraint (5.12).

Finally, putting everything together, the full hamiltonian is,

$$
\begin{aligned}
H= & \frac{1}{2} \sum_{\sigma, \sigma^{\prime}} G\left(\sigma, \sigma^{\prime}\right)\left(\frac{m_{r}^{2}}{2} \rho(\sigma) \rho\left(\sigma^{\prime}\right)+\rho\left(\sigma^{\prime}\right) \int d \mathbf{q} \mathbf{q}^{2}\left(\phi_{1}^{\dagger} \phi_{1}\right)_{\sigma, \mathbf{q}}\right. \\
& \left.-\int d \mathbf{q} \int d \mathbf{q}^{\prime}\left(\mathbf{q} \cdot \mathbf{q}^{\prime}\right)\left(\phi_{0}\left(\phi_{1}^{\dagger}+\phi_{1}\right)+\phi_{1}^{\dagger} \phi_{1}\right)_{\sigma, \mathbf{q}}\left(\phi_{0}\left(\phi_{1}^{\dagger}+\phi_{1}\right)+\phi_{1}^{\dagger} \phi_{1}\right)_{\sigma^{\prime}, \mathbf{q}^{\prime}}\right) \\
& +\sum_{\sigma} \lambda_{0}(\sigma) \int d \mathbf{q}\left(\phi_{1}^{\dagger} \phi_{1}\right)_{\sigma, \mathbf{q}},
\end{aligned}
$$

supplemented by the constraint (5.12). As explained earlier, the classical part of $H$ is absorbed into mass renormalization. Also since $\phi_{0}$ and $\lambda_{0}$ satisfy the equations of motion, linear terms in $\phi_{1}$ and $\phi_{1}^{\dagger}$ are absent. 


\section{The continuum limit}

In this section, we will take the continuum limit on the world sheet, by letting a, the spacing in the $\sigma$ direction go to zero. First consider the kinetic energy term in the action (3.12):

$$
S_{k . e}=2 \int d \tau \sum_{\sigma} \int d \mathbf{q} \phi_{1, i} \partial_{\tau} \phi_{1, r}
$$

where $\phi_{r}$ and $\phi_{i}$ are the real and imaginary components of $\phi$. In making transition to the continuum limit by

$$
\sum_{\sigma} \rightarrow \frac{1}{a} \int_{0}^{p^{+}} d \sigma,
$$

an extra factor of $1 / a$ is introduced, which has to be scaled away in order to have a canonically normalized kinetic energy term. $\phi_{0}$, which is real and therefore part of $\phi_{r}$, is independent of $a$ (eq. (5.10)). If we want the classical solution to survive in $a \rightarrow 0$ limit, $\phi_{r}$ cannot be scaled by $a$. Therefore, the only remaining possibility is to scale $\phi_{i}$ by $a$. For convenience, we redefine $\phi_{i}$ by letting

$$
\phi_{i} \rightarrow a \phi_{i}
$$

and replace in (6.1) the sum over $\sigma$ by an integral:

$$
S_{k . e}=2 \int d \tau \int_{0}^{p^{+}} d \sigma \int d \mathbf{q} \phi_{i} \partial_{\tau} \phi_{r}=2 \int d \tau \int_{0}^{p^{+}} d \sigma \int d \mathbf{q} \phi_{1, i} \partial_{\tau}\left(\phi_{0}+\phi_{1, r}\right) .
$$

Notice that $\phi_{i}=\phi_{1, i}$ since $\phi_{0}$ is real.

Next we would like to derive the continuum limit of the hamiltonian (5.13). It will consist of two terms: One proportional to $1 / a^{2}$ the other independent of $a$,

$$
H=\frac{1}{a^{2}} H^{(1)}+H^{(2)} .
$$

Terms proportional to positive powers of $a$ will be dropped since they vanish in the continuum limit. The main problem is the factor $\mathcal{E}$ in the definition of $G$ (eq. (3.6)), which is so far defined only for a discretized $\sigma$ coordinate. Consider an interval of $\sigma$ much bigger than the lattice spacing a:

$$
\Delta \sigma=\sigma_{j}-\sigma_{i} \gg a,
$$

so that, as $a \rightarrow 0, \Delta \sigma$ is kept fixed. If $\rho$ is different from zero in this interval, $\mathcal{E}\left(\sigma_{i}, \sigma_{i}+\Delta \sigma\right)$, which has $n$ factors of $1-\rho$ in its definition, where

$$
n=\frac{\Delta \sigma}{a}-1,
$$

rapidly goes to zero as $a \rightarrow 0, n \rightarrow \infty$. Then, in this limit, assuming that $\rho \neq 0$, the sums over $\sigma$ and $\sigma^{\prime}$ in eq. (5.13) have contribution only from very small $\left|\sigma-\sigma^{\prime}\right|$ of the order of a. We will call this phenomenon localization: As lattice spacing $a$ goes to zero, $\sigma \rightarrow \sigma^{\prime}$, and the non-local terms in the hamiltonian approach a local limit. This result is based on two assumptions: 
a) Generically, $\rho$ is different from zero. Since, by its definition (eq. (3.5)), $\rho$ measures the density of solid lines on the world sheet, we are assuming that the world sheet is densely populated with Feynman graphs. Later, we will justify this assumption by showing that one can expand $\rho$ about a background $\rho_{0} \neq 0$.

b) $\phi$ and $\rho$ are slowly varying fields. More precisely, this means that the typical length scale that governs the variation of these fields is not $a$ but, as we shall see later, it is $g$, the coupling constant, which has the dimension of mass and is independent of $a$.

A typical term involving $G$ in $H$ is of the form

$$
H \simeq \frac{1}{2} \sum_{\sigma, \sigma^{\prime}} G\left(\sigma, \sigma^{\prime}\right) A(\sigma) B\left(\sigma^{\prime}\right) .
$$

Under the assumption of localization, it is natural to expand the factor $A B$ in powers of $\sigma^{\prime}-\sigma$ :

$$
A(\sigma) B\left(\sigma^{\prime}\right)=A(\sigma) B(\sigma)+\left(\sigma^{\prime}-\sigma\right) A(\sigma) B^{\prime \prime}(\sigma)+\frac{1}{2}\left(\sigma^{\prime}-\sigma\right)^{2} A(\sigma) B^{\prime \prime}(\sigma)+\cdots,
$$

where primes indicate derivatives with respect to $\sigma$. We have stopped at the second order in this expansion; we shall shortly see that each power of $\sigma^{\prime}-\sigma$ corresponds to a power of $a$, and terms higher than second order do not contribute in the continuum limit. Plugging in the above expansion in (5.13), we encounter the sums

$$
\begin{aligned}
\sum_{\sigma^{\prime}} G\left(\sigma, \sigma^{\prime}\right)=2 \sum_{n=0}^{\infty} \frac{(1-\rho)^{n}}{a(n+1)} & =\frac{2}{a} L(\sigma) \\
\sum_{\sigma^{\prime}}\left(\sigma^{\prime}-\sigma\right) G\left(\sigma, \sigma^{\prime}\right) & =0 \\
\sum_{\sigma^{\prime}}\left(\sigma^{\prime}-\sigma\right)^{2} G\left(\sigma, \sigma^{\prime}\right) & =2 a \sum_{n=0}^{\infty}(n+1)(1-\rho)^{n}=\frac{2 a}{\rho^{2}},
\end{aligned}
$$

where,

$$
L=-\frac{\ln (\rho(\sigma))}{1-\rho(\sigma)} .
$$

In carrying out these sums, we have taken $\rho$ to be $\sigma$ independent. Actually, a weaker condition of slowly varying $\rho(\sigma)$ is sufficient, provided that we also expand $G$ up to second order in $\sigma^{\prime}-\sigma$. This amounts to expanding various fields up to second order in derivatives with respect to $\sigma$ around a slowly varying background. A typical term in the hamiltonian can then be cast into the following form:

$$
\begin{gathered}
H \rightarrow \int_{0}^{p^{+}} d \sigma\left(\left(\frac{L(\sigma)}{a^{2}}+f_{1}(\rho(\sigma))\left(\rho^{\prime}(\sigma)\right)^{2}+f_{2}(\rho(\sigma)) \rho^{\prime \prime}(\sigma)\right) A(\sigma) B(\sigma)\right. \\
\left.-\frac{1}{2 \rho^{2}(\sigma)} A^{\prime}(\sigma) B^{\prime}(\sigma)\right),
\end{gathered}
$$

where we have converted the sum over $\sigma$ into an integral by (6.2). $f_{1,2}$ are local functions of $\rho$ which can be computed by expanding $G$ in powers of $\sigma^{\prime}-\sigma$. Since they will not be needed in the subsequent development, we have not written them out explicitly. 
We note that $H$ has one term that goes as $1 / a^{2}$, and other terms that are $a$ independent. Also, it is local, with at most two derivatives in $\sigma$ and in $\tau$. It is gratifying that the $a \rightarrow 0$ limit produces a local action of the standard canonical type on the world sheet.

Eq. (6.7) can now be used directly in the hamiltonian to find the continuum limit. This results in a rather lengthy expression. In the next section, we shall see that for the sector of the model we are interested in, the light sector, there is a great simplification; so instead, we will carry out the computation for only this sector.

\section{The light sector of the model}

A crucial feature of eq. (6.8) is the first term, which blows up as $a \rightarrow 0$. Since the kinetic energy term (6.3) remains finite in this limit, this implies that masses of various modes go to infinity. As was done in reference [1], one can show this explicitly by expanding to quadratic order around a fixed background in $\rho=\rho_{0}$. In any case, this result is pretty obvious without any calculation. The important question is whether there are any "light" modes, whose masses stay finite as $a \rightarrow 0$. In what follows, we will indeed identify two such modes. We will then assume that the "heavy" modes will decouple in continuum limit, and only the light sector will remain behind. Although we have no rigorous proof of it, it is a hypothesis very commonly used in field theory.

One of the two light modes is $\rho$ itself. Its mass comes from the first term in eq. (5.13):

$$
H_{m \rho}=\frac{m_{r}^{2}}{4} \sum_{\sigma, \sigma^{\prime}} G\left(\sigma, \sigma^{\prime}\right) \rho(\sigma) \rho\left(\sigma^{\prime}\right) \rightarrow \frac{m_{r}^{2}}{2 a^{2}} \int_{0}^{p^{+}} d \sigma L(\sigma) \rho^{2}(\sigma) .
$$

We have taken the continuum limit using (6.7) and kept the $1 / a^{2}$ term. The mass comes from expanding it around a fixed background $\rho_{0}$. Clearly, to have a finite mass as $a \rightarrow 0$, $m_{r}$ must be scaled as

$$
m_{r} \rightarrow a u \text {. }
$$

The parameter $u$ is dimensionless. This may seem surprising; however, after the coefficient of the kinetic energy term is normalized, the mass of $\rho$ will turn out to be proportional to $g u$ (eq. (8.5)), which has the correct dimension since $g$ has dimension of mass. The above scaling is therefore necessary to have the mass term with the correct dimension. In contrast, $g$ needs no scaling: It already has the correct dimension to be the coupling constant of the $\phi^{3}$ theory in four dimensions.

To finish this part of the story, we add an external source to $H_{m \rho}$, which is really the potential term for $\rho$ :

$$
H_{m \rho} \rightarrow \int_{0}^{p^{+}} d \sigma\left(-J \rho+\frac{1}{2} u^{2} L \rho^{2}\right)
$$

where $L$ is given by (6.7), and $J$ is a $\sigma, \tau$ independent external field (constant background). $J$ can be arranged to produce a minimum at some constant $\rho=\rho_{0}$, satisfying $0<\rho_{0}<1$, around which $\rho$ can be expanded by setting

$$
\rho=\rho_{0}+\rho_{1}
$$


and expanding in $\rho_{1}$.

The second light mode is related to translation invariance in momentum space (eq. (3.13)). This invariance is broken spontaneously by the classical solution $\phi_{0}$, which is not translation invariant. This situation is familiar from soliton and instanton physics; as a consequence of Goldstone's theorem, a massless zero mode develops. Protected by Goldstone's theorem, this mode stays massless also in the limit $a \rightarrow 0$, and therefore it belongs to the light sector. We will identify and quantize this mode by means of the collective coordinate method, widely used for quantizing in a soliton background.

Consider the field configuration

$$
\phi_{r} \rightarrow \phi_{0}(\mathbf{q}+\mathbf{v})=-g \frac{(\rho(\sigma, \tau))^{1 / 2}}{(\mathbf{q}+\mathbf{v}(\sigma, \tau))^{2}+\pi g^{2}} .
$$

What we have done is to promote the constant vector $\mathbf{r}$ into the field $\mathbf{v}(\sigma, \tau)$ on the world sheet. In the action, we will therefore make the ansatz

$$
\phi_{1, r} \rightarrow \phi_{0}(\mathbf{q}+\mathbf{v})-\phi_{0}(\mathbf{q}) .
$$

On the other hand, we will keep $\phi_{1, i}$ arbitrary, since in the first order formalism for the action (eq. (6.3)), $\phi_{r}$ and $\phi_{i}$ cannot be simultaneously fixed. However, we note that since $\phi_{i}$ has been scaled by a factor of $a$, higher order terms in $\phi_{i}$, unless accompanied by compansating factors of $1 / a$, can be dropped in the limit $a \rightarrow 0$.

We can now replace $\phi_{1, r}$ by (7.5), and then take the continuum limit of $\mathrm{H}$ using (6.7). Instead, it is possible to simplify the computation drastically and also understand the simplicity of the final result by the following observation: Translation invariance (3.13) means that the resulting action is invariant under the shift

$$
\mathbf{v} \rightarrow \mathbf{v}+\mathbf{r}
$$

where $\mathbf{r}$ is again a constant vector. This not only forbids a mass term for $\mathbf{v}$, but also a term such as

$$
\left(\partial_{\sigma, \tau} \mathbf{v}\right)^{2}(\mathbf{v})^{2}
$$

is not allowed. To eliminate these terms, one can treat $\mathbf{v}, \partial_{\sigma} \mathbf{v}$ and $\partial_{\tau} \mathbf{v}$ as independent variables at fixed $\sigma$ and $\tau$, and set $\mathbf{v}=0$, while keeping $\partial_{\sigma, \tau} \mathbf{v}$, and also $\rho$ non-zero. Of course, a direct calculation would give the same result, and what we are doing is to take a shortcut.

Let us now see what happens to some of these terms in (5.13) after this simplification. Consider the third term on the right: With the identification

$$
\begin{aligned}
\mathbf{A}=-\mathbf{B} & =\int d \mathbf{q} \mathbf{q}\left(\phi_{0}\left(\phi_{1}^{\dagger}+\phi_{1}\right)+\phi_{1}^{\dagger} \phi_{1}\right)=\int d \mathbf{q} \mathbf{q}\left(2 \phi_{0} \phi_{1, r}+\phi_{1, r}^{2}+a^{2} \phi_{1, i}^{2}\right) \\
& \rightarrow \int d \mathbf{q} \mathbf{q}\left(\phi_{0}^{2}(\mathbf{q}+\mathbf{v})-\phi_{0}^{2}(\mathbf{q})+a^{2} \phi_{1, i}^{2}\right),
\end{aligned}
$$

we can apply eq. (6.7). The first term, with no $\sigma$ derivatives, vanishes:

$$
\phi_{0}^{2}(\mathbf{q}+\mathbf{v})-\phi_{0}^{2}(\mathbf{q}) \rightarrow 0
$$


as $\mathbf{v} \rightarrow 0$. Also, $a^{2} \phi_{1, i}^{2}$ can be dropped as $a \rightarrow 0$. Therefore, only the last term in (6.7) with sigma derivatives survives. In this term, as explained above, we set $\mathbf{v}=0$, while keeping $\partial_{\sigma} \mathbf{v}$ non-zero. With these simplifications, we have, in the limit $a \rightarrow 0$ and $\mathbf{v} \rightarrow 0$,

$$
\partial_{\sigma} \mathbf{A} \rightarrow 4 g^{2} \rho \int d \mathbf{q} \mathbf{q} \frac{\mathbf{q} \cdot \partial_{\sigma} \mathbf{v}}{\left(\mathbf{q}^{2}+\pi g^{2}\right)^{3}}=\rho \partial_{\sigma} \mathbf{v}
$$

and therefore,

$$
\frac{1}{2} \sum_{\sigma, \sigma^{\prime}} G\left(\sigma, \sigma^{\prime}\right) \mathbf{A}(\sigma) \cdot \mathbf{B}\left(\sigma^{\prime}\right) \rightarrow \frac{1}{2} \int_{0}^{p^{+}} d \sigma\left(\partial_{\sigma} \mathbf{v}\right)^{2} .
$$

Now consider the second and the last terms on the right in (5.13). Both of these terms contain the factor

$$
\phi_{1}^{\dagger} \phi_{1} \rightarrow\left(\phi_{0}(\mathbf{q}+\mathbf{v})-\phi_{0}(\mathbf{q})\right)^{2}+a^{2} \phi_{1, i}^{2} .
$$

As $\mathbf{v} \rightarrow 0$, the first term vanishes. We cannot, however, drop the second term as $a \rightarrow 0$, since there is a factor $1 / a^{2}$ multiplying it. To see this, we rewrite these terms as

$$
\begin{gathered}
\frac{1}{2} \sum_{\sigma, \sigma^{\prime}} G\left(\sigma, \sigma^{\prime}\right) \rho\left(\sigma^{\prime}\right) \int d \mathbf{q} \mathbf{q}^{2}\left(\phi_{1}^{\dagger} \phi_{1}\right)_{\sigma, \mathbf{q}}+\sum_{\sigma} \lambda_{0}(\sigma) \int d \mathbf{q}\left(\phi_{1}^{\dagger} \phi_{1}\right)_{\sigma, \mathbf{q}} \\
\rightarrow \frac{1}{2} \sum_{\sigma, \sigma^{\prime}} G\left(\sigma, \sigma^{\prime}\right) \rho\left(\sigma^{\prime}\right) \int d \mathbf{q}\left(\mathbf{q}^{2}+\pi g^{2}\right) a^{2} \phi_{1, i}^{2}\left(\sigma^{\prime}, \mathbf{q}\right) \\
\rightarrow \int_{0}^{p^{+}} d \sigma \int d \mathbf{q} L(\sigma) \rho(\sigma)\left(\mathbf{q}^{2}+\pi g^{2}\right) \phi_{1, i}^{2}(\sigma, \mathbf{q}),
\end{gathered}
$$

where we have used,

$$
\lambda_{0}=\pi g^{2} E=\frac{\pi g^{2}}{2} \sum_{\sigma^{\prime}} G\left(\sigma, \sigma^{\prime}\right) \rho\left(\sigma^{\prime}\right) \rightarrow \frac{\pi g^{2}}{a} L(\sigma) \rho(\sigma),
$$

which follows from (6.7). The above factor of $1 / a$, combined with an additional $1 / a$ coming from converting the sum over $\sigma$ into an integral, cancels the factor of $a^{2}$ multiplying $\phi_{1, i}^{2}$, so the final result is $a$ independent.

Putting together (7.10) and (7.12), the hamiltonian for the light sector is

$$
H=H_{m \rho}+\int_{0}^{p^{+}} d \sigma \int d \mathbf{q} L(\sigma) \rho(\sigma)\left(\mathbf{q}^{2}+\pi g^{2}\right) \phi_{1, i}^{2}(\sigma, \mathbf{q})+\frac{1}{2} \int_{0}^{p^{+}} d \sigma\left(\partial_{\sigma} \mathbf{v}\right)^{2} .
$$

Notice that the terms proportional to $1 / a^{2}$ in the hamiltonian have disappeared: the light sector is really light.

The above hamiltonian has to be supplemented by the constraint (5.12). This presents no problem: in the limit $a \rightarrow 0$, the ansatz (7.6) automatically satisfies this constraint:

$$
\int d \mathbf{q}\left(\phi_{0}\left(\phi_{1}+\phi_{1}^{\dagger}\right)+\phi_{1}^{\dagger} \phi_{1}\right) \stackrel{a \rightarrow 0}{\rightarrow} \int d \mathbf{q}\left(\phi_{0}^{2}(\mathbf{q}+\mathbf{v})-\phi_{0}^{2}(\mathbf{q})\right)=0 .
$$

One remaining question is whether there are additional light modes we might have missed. In reference [1], by expanding to quadratic order around a constant background, it was shown that the masses of all the other modes go as $1 / a^{2}$ as $a \rightarrow 0$. 


\section{The light sector action and string formation}

In the previous section, we presented the hamiltonian (7.13) for the light modes. The corresponding action is given by (6.3). We need

$$
\partial_{\tau} \phi_{r} \rightarrow \partial_{\tau} \phi_{0}(\sigma, \tau, \mathbf{q}+\mathbf{v}) \stackrel{\mathbf{v} \rightarrow 0}{\rightarrow}-\frac{g \partial_{\tau} \rho}{2 \sqrt{\rho}\left(\mathbf{q}^{2}+\pi g^{2}\right)}+\frac{2 g \sqrt{\rho} \mathbf{q} \cdot \partial_{\tau} \mathbf{v}}{\left(\mathbf{q}^{2}+\pi g^{2}\right)^{2}}
$$

The resulting expression is at most quadratic in $\phi_{1, i}$. This field can then be eliminated by doing the gaussian functional integral over it, which amounts to solving its equation of motion. The result is

$$
\begin{aligned}
S & =\int d \tau \int_{0}^{p^{+}} d \sigma\left(\int d \mathbf{q} \frac{g^{2}\left(\frac{2 \rho^{1 / 2} \mathbf{q} \cdot \partial_{\tau} \mathbf{v}}{\left(\mathbf{q}^{2}+\pi g^{2}\right)^{2}}-\frac{\partial_{\tau} \rho}{2 \rho^{1 / 2}\left(\mathbf{q}^{2}+\pi g^{2}\right)}\right)^{2}}{\rho L\left(\mathbf{q}^{2}+\pi g^{2}\right)}-\frac{1}{2} u^{2} \rho^{2} L+J \rho\right) \\
& =\int d \tau \int_{0}^{p^{+}} d \sigma\left(\frac{\left(\partial_{\tau} \mathbf{v}\right)^{2}}{6 \pi^{2} g^{4} L}-\frac{1}{2}\left(\partial_{\sigma} \mathbf{v}\right)^{2}+\frac{\left(\partial_{\tau} \rho\right)^{2}}{8 \pi g^{2} \rho^{2} L}-\frac{1}{2} u^{2} \rho^{2} L+J \rho\right) .
\end{aligned}
$$

The above world sheet action is the fundamental result of the present paper. There is no longer any reference to the target space except for the number of components of $\mathbf{v}$; the field $\phi$, as well as the momentum $\mathbf{q}$ and the cutoff "a" have all disappeared from the problem. This great simplification is the result of the decoupling of heavy modes and of localization on the world sheet in the limit $a \rightarrow 0$.

In deriving this result, so far there have been no approximations. The only hypothesis that was needed was the decoupling of the heavy states in the limit $a \rightarrow 0$. However, this action, which has inverse powers of $\rho$, is meaningful only if it is expanded around a no-zero value of $\rho$. We note that at $\rho_{0}=0$, the background (eq. (5.10)) around which we are expanding vanishes, and many of the steps leading to (8.2) which relied on this background no longer go through. Therefore, a non-zero $\rho_{0}$ is essential for the derivation of eq. (8.2). The starting point, the usual graph expansion, was based on the phase $\rho_{0}=0$. In contrast, as we shall see, in the phase $\rho_{0} \neq 0$, a string is formed, and the coupling constant is absorbed into the definition of the string slope (eq. (8.6)).

The natural choice for the constant $\rho_{0}$ is the minimum of the potential in eq. (7.3). We can now expand $\rho$ as in eq. (7.4) abot this minimum. The quadratic terms in this expansion are of particular interest:

$$
\begin{aligned}
S_{2} & =\int d \tau \int_{0}^{p^{+}} d \sigma\left(\frac{\left(\partial_{\tau} \mathbf{v}\right)^{2}}{6 \pi^{2} g^{4} L\left(\rho_{0}\right)}-\frac{1}{2}\left(\partial_{\sigma} \mathbf{v}\right)^{2}+\frac{\left(\partial_{\tau} \rho_{1}\right)^{2}}{8 \pi g^{2} \rho_{0}^{2} L\left(\rho_{0}\right)}-\frac{1}{2} z^{2}\left(\rho_{0}\right) \rho_{1}^{2}\right) \\
& \rightarrow \int d \tau \int_{0}^{p^{+}} d \sigma\left(\frac{1}{2}\left(\partial_{\tau} \tilde{\mathbf{v}}\right)^{2}-3 \pi^{2} g^{4} L\left(\rho_{0}\right)\left(\partial_{\sigma} \tilde{\mathbf{v}}\right)^{2}+\frac{1}{2}\left(\partial_{\tau} \tilde{\rho}_{1}\right)^{2}-\frac{1}{2} \mu^{2}\left(\rho_{0}\right) \tilde{\rho}_{1}^{2}\right),
\end{aligned}
$$

where we have scaled the fields by

$$
\mathbf{v}=\sqrt{3 \pi^{2} g^{4} L} \tilde{\mathbf{v}}, \quad \rho_{1}=\sqrt{4 \pi g^{2} \rho_{0}^{2} L} \tilde{\rho}_{1}
$$


This is the world sheet action for a massive field $\tilde{\rho_{1}}$ plus a string generated by $\tilde{\mathbf{v}}$. The mass $\mu$ of $\tilde{\rho_{1}}$ is given by

$$
\mu^{2}=4 \pi g^{2} u^{2} L\left(\rho_{0}\right) \rho_{0}^{2} z^{2}, z^{2}=\frac{3-4 \rho_{0}+\rho_{0}^{2}+2 \ln \left(\rho_{0}\right)}{2\left(\rho_{0}-1\right)^{3}} .
$$

$\mu^{2}$ is positive in the physical range $0<\rho_{0}<1$. The string slope is

$$
\alpha^{\prime}=\left(12 \pi^{4} g^{4} L\left(\rho_{0}\right)\right)^{1 / 2} .
$$

The dimensions of both the $\rho_{1}$ mass and the string slope are provided by the coupling constant $g$, the only dimensional parameter in the problem in the absence of $a$.

We note that, if we set $\rho_{1}=0$, we end up with a standard free string in the light cone frame [10]. This is because translation invariance (7.7) does not allow any higher order interaction terms involving $\mathbf{v}$ alone. These non-linear terms are however induced through the coupling between $\rho$ and $\mathbf{v}$ in the full action. Also, although there is no $\partial_{\sigma} \rho$ term in the action, it will be generated as a higher order contribution. To avoid confusion, we emphasize that since we are summing the planar graphs, the string is always a free string. String interactions can only come from the non-planar graphs. What is happening is that with $\rho$ couplings included, the string becomes non-linear: The straight trajectories of the linear string are replaced by curved trajectories.

Superficially, it might appear that the expansion discussed above is the same as expanding in powers of $g$. Howevever, this is not quite right; in the limit $g \rightarrow 0$, both the string slope and the $\rho$ mass vanish. From the string perspective, this is a singular limit, so it is better to trade $g$ for the string slope and give up on expanding in powers of $g$. The situation here is similar to the Higgs effect, when one of the coupling constants is transmuted into a mass parameter. What we have here are two complementary pictures: In the limit $g \rightarrow 0$, the appropriate picture is the usual field theory picture of graph expansion. String picture makes sense only for a world sheet densely covered by graphs, which is clearly requies $g \neq 0$, since otherwise higher order graphs needed to cover the world sheet would be suppressed.

\section{$9 \quad$ Four transverse dimensions}

In this section, we will discuss the $D=4$ case, mostly pointing out the changes that have to be made in going from $D=2$ to $D=4$. The development all the way to section 5 , eq. (5.3), is independent of the number of dimensions and remains unchanged. The first change occurs in the calculation of the classical action $S_{c}$ (eq. (5.4)), which is now quadratically divergent:

$$
\begin{aligned}
S_{c} & \simeq-g \int d \tau \sum_{\sigma} W(\sigma, \tau) \int d \mathbf{q} \phi_{0}(\sigma, \tau, \mathbf{q})=g^{2} \int d \tau \sum_{\sigma} \int d \mathbf{q} \frac{W^{2}}{E \mathbf{q}^{2}+\lambda} \\
& \simeq g^{2} \pi^{2} \Lambda^{2} \int d \tau \sum_{\sigma} \frac{W^{2}}{E} .
\end{aligned}
$$


Here $\Lambda$ is the ultraviolet cutoff and we have kept the leading quadratic divergence. The only difference between this and eq. (5.4) is that a log divergence is replaced by a quadratic one. As before, requiring it be proportional to the mass term in (5.13) for renormalizability, we again get exactly eq. (5.6) for W. Also splitting $\lambda$ as in eq. (5.7), eq. (5.8) for $\phi_{0}$ remains unchanged.

To determine $\lambda_{0}$, we again impose the constraint (3.4), which now involves a logarithmically divergent integral. The result is

$$
\frac{1}{g_{0}^{2}}=\pi^{2} \int_{0}^{\Lambda^{2}} d \mathbf{q}^{2} \frac{\mathbf{q}^{2}}{\left(\mathbf{q}^{2}+\lambda_{0} / E\right)^{2}}=\pi^{2}\left(\ln \left(\frac{E}{\lambda_{0}} \Lambda^{2}\right)-1\right),
$$

where we have replaced $g$ by $g_{0}$ to make it clear that we are dealing with the bare coupling constant. Since $g_{0}$ is $\sigma, \tau$ independent, it follows that

$$
\lambda_{0}(\sigma, \tau)=\mu_{0}^{2} E(\sigma, \tau)
$$

where $\mu_{0}$ is a $\sigma, \tau$ independent constant and eq. (9.2) can be rewritten as

$$
\frac{1}{g_{0}^{2}}=\pi^{2}\left(\ln \left(\Lambda^{2} / \mu_{0}^{2}\right)-1\right)
$$

We will determine $\mu_{0}$ later on in terms of the slope of the string; all we need to know at the moment is that

$$
\Lambda / \mu_{0} \rightarrow \infty
$$

as $\Lambda \rightarrow \infty$. Putting together these changes, we have the result

$$
\phi_{0}=-g_{0} \frac{\sqrt{\rho}}{\mathbf{q}^{2}+\mu_{0}^{2}} .
$$

From this point on, till eq. (7.9) in section 7 , there are no significant changes except for the replacement

$$
\pi g^{2} \rightarrow \mu_{0}^{2}
$$

in the expression for $\phi_{0}$. However, in eq. (7.9), the integral is over four dimensions and it is logarithmically divergent. This equation now reads

$$
\begin{aligned}
\partial_{\sigma} \mathbf{A} & \rightarrow 4 g^{2} \rho \int d \mathbf{q} \mathbf{q} \frac{\mathbf{q} \cdot \partial_{\sigma} \mathbf{v}}{\left(\mathbf{q}^{2}+\mu_{0}^{2}\right)^{3}} \\
& =g_{0}^{2} \pi^{2} \ln \left(\frac{\Lambda^{2}}{\mu_{0}^{2}}\right) \partial_{\sigma} \mathbf{v} \rightarrow \rho \partial_{\sigma} \mathbf{v} .
\end{aligned}
$$

In the last step, using eq. (9.4) for $g_{0}$, the log divergence cancelled. The final result is identical to $D=2$ case, and eq. (7.10) remains unchanged.

Next, we turn to the eq. (8.2). The coefficient of $\left(\partial_{\sigma} \mathbf{v}\right)^{2}$ is unchanged, but the coefficients of $\left(\partial_{\tau} \mathbf{v}\right)^{2}$ and $\left(\partial_{\tau} \rho\right)^{2}$ will be different. The differences come from the replacement $\pi g^{2} \rightarrow \mu_{0}^{2}$ and from the change of the dimension of integration. The integrals in (8.2) are convergent at $D=4$, and the resulting action is

$$
S=\int d \tau \int_{0}^{p^{+}} d \sigma\left(\frac{\pi^{2} g_{0}^{2}}{12 L \mu_{0}^{4}}\left(\partial_{\tau} \mathbf{v}\right)^{2}-\frac{1}{2}\left(\partial_{\sigma} \mathbf{v}\right)^{2}+\frac{\pi^{2} g_{0}^{2}}{8 \rho^{2} L \mu_{0}^{2}}\left(\partial_{\tau} \rho\right)^{2}-\frac{1}{2} u^{2} \rho^{2} L+J \rho\right) .
$$


Expanding around $\rho=\rho_{0}$ as before, we again get a linear string plus a massive $\rho$ field. The string slope is given by

$$
\alpha^{\prime}=\left(\frac{24 L\left(\rho_{0}\right) \mu_{0}^{4}}{g_{0}^{2}}\right)^{1 / 2}
$$

or,

$$
\frac{\mu_{0}^{2}}{\alpha^{\prime}}=\left(24 \pi^{2} L\left(\rho_{0}\right)\left(\ln \left(\Lambda^{2} / \alpha^{\prime}\right)-\ln \left(\mu_{0}^{2} / \alpha^{\prime}\right)\right)\right)^{-1 / 2} .
$$

The model is renormalized by keeping the physical parameter $\alpha^{\prime}$ fixed as $\Lambda \rightarrow \infty$, and solving for $\mu_{0}$ and $g_{0}$ in terms of $\alpha^{\prime}$ and $\lambda$. To leading order in $\Lambda$,

$$
\frac{\mu_{0}^{2}}{\alpha^{\prime}} \rightarrow\left(24 \pi^{2} L\left(\rho_{0}\right) \ln \left(\Lambda^{2} / \alpha^{\prime}\right)\right)^{-1 / 2},
$$

and,

$$
\frac{1}{g_{0}^{2}} \rightarrow \pi^{2} \ln \left(\Lambda^{2} / \alpha^{\prime}\right)
$$

as $\Lambda \rightarrow \infty . \phi^{3}$ is known to be asymptotically free in $5+1$ dimensions, and this dependence of the bare coupling constant on the cutoff is characteristic of such a theory. We note that the contribution of $\mu_{0}^{2}$ to $1 / g_{0}^{2}$ goes as $\left.\ln (\ln \Lambda)\right)$ and it is negligible compared to the leading $\ln (\Lambda)$ dependence.

So, despite the complications caused by the cutoff dependent coupling constant, the string sector at $D=4$ works out pretty much as at $D=2$ after slope renormalization. The $\rho$ sector of the model is, however, more problematic. In the limit of large $\Lambda$, the coefficient of the kinetic energy term for $\rho$ goes to zero as

$$
\frac{g_{0}^{2}}{\mu_{0}^{2}} \rightarrow\left(\ln \left(\Lambda^{2} / \alpha^{\prime}\right)\right)^{-1 / 2}
$$

Again expanding about $\rho=\rho_{0}$ and keeping only quadratic terms, we have a simple harmonic oscillator at each value of $\sigma$, decoupled from the others. For the usual SHO lagrangian

$$
\mathcal{L}=\frac{1}{2} m \dot{x}^{2}-\frac{1}{2} k x^{2}
$$

the limit we are considering corresponds to $m \rightarrow 0$. In the zero mass limit, the energies of all the excited states go to infinity, and after subtracting the zero point energy, we are left with only the ground state at zero energy. Therefore, $\rho_{1}$ collapses into time independent projection operators:

$$
\rho_{1}(\sigma) \rightarrow|0\rangle_{\sigma}\left\langle\left. 0\right|_{\sigma}\right.
$$

where $|0\rangle_{\sigma}$ are ground states, one for each $\sigma$, that are mutually orthogonal. Further investigation, which we will not undertake here, is necessary to verify that the interaction terms do not change this picture.

The foregoing discussion is incomplete in one important respect: we have tacitly assumed that the one loop result (9.9) for the coupling constant holds all the way from energies of the order of $\Lambda$ to the string slope $\alpha^{\prime}$. Of course, what is needed is a renormalization group analysis for the running coupling constant. We leave this interesting problem for future research. 


\section{Conclusions}

The main result of the present article is the derivation of world sheet action given by eqs. (8.2) and (9.7). The main advance made on reference [1] is that these actions are now defined on a continuously parametrized world sheet and the cutoff parameter "a" which discretized the world sheet has disappeared. There is also now an additional dynamical variable $\rho$; expanding $\rho$ around the minimum of its potential, we recover a linear string, but there are also fluctuations around this minimum which signal deviations from a linear string.

There are still important problems left for future research. One of them is the question of Lorentz invariance. ${ }^{1}$ Although we have not done so here, it is not difficult to establish invariance under the so called light cone Galilean subgroup of the Lorentz group, but full Lorentz invariance is still an open question. Another important target for research is to extend world sheet methods to more physical theories. Although some initial attempts were made in this direction [13-15], much still remains to be done.

\section{Acknowledgments}

This work was supported in part by the Director, Office of Science, Office of High Energy Physics of the U.S. Department of Energy under the Contract DE-AC02-05CH11231.

Open Access. This article is distributed under the terms of the Creative Commons Attribution Noncommercial License which permits any noncommercial use, distribution, and reproduction in any medium, provided the original author(s) and source are credited.

\section{References}

[1] K. Bardakci, Mean field method applied to the new world sheet field theory: string formation, JHEP 03 (2009) 088 [arXiv:0901.0949] [SPIRES].

[2] K. Bardakci, A new world sheet field theory, JHEP 10 (2008) 056 [arXiv:0808.2959] [SPIRES].

[3] K. Bardakci and C.B. Thorn, A worldsheet description of large- $N_{c}$ quantum field theory, Nucl. Phys. B 626 (2002) 287 [hep-th/0110301] [SPIRES].

[4] G. 't Hooft, A planar diagram theory for strong interactions, Nucl. Phys. B 72 (1974) 461 [SPIRES].

[5] H.B. Nielsen and P. Olesen, A parton view on dual amplitudes, Phys. Lett. B 32 (1970) 203 [SPIRES].

[6] B. Sakita and M.A. Virasoro, Dynamical model of dual amplitudes, Phys. Rev. Lett. 24 (1970) 1146.

[7] A. Casher, Gauge fields on the null plane, Phys. Rev. D 14 (1976) 452 [SPIRES].

[8] R. Giles and C.B. Thorn, A lattice approach to string theory, Phys. Rev. D 16 (1977) 366 [SPIRES].

\footnotetext{
${ }^{1}$ See $[11,12]$ for an investigation of renormalization and Lorentz invariance in the light cone formulation.
} 
[9] T. Banks, W. Fischler, S.H. Shenker and L. Susskind, $M$ theory as a matrix model: a conjecture, Phys. Rev. D 55 (1997) 5112 [hep-th/9610043] [SPIRES].

[10] P. Goddard, J. Goldstone, C. Rebbi and C.B. Thorn, Quantum dynamics of a massless relativistic string, Nucl. Phys. B 56 (1973) 109 [SPIRES].

[11] C.B. Thorn, Renormalization of quantum fields on the lightcone worldsheet. I: scalar fields, Nucl. Phys. B 699 (2004) 427 [hep-th/0405018] [SPIRES].

[12] D. Chakrabarti, J. Qiu and C.B. Thorn, Scattering of glue by glue on the light-cone worldsheet. II: helicity conserving amplitudes, Phys. Rev. D 74 (2006) 045018 [hep-th/0602026] [SPIRES].

[13] C.B. Thorn, A worldsheet description of planar Yang-Mills theory, Nucl. Phys. B 637 (2002) 272 [hep-th/0203167] [SPIRES].

[14] S. Gudmundsson, C.B. Thorn and T.A. Tran, BT worldsheet for supersymmetric gauge theories, Nucl. Phys. B 649 (2003) 3 [hep-th/0209102] [SPIRES].

[15] C.B. Thorn and T.A. Tran, The fishnet as anti-ferromagnetic phase of worldsheet Ising spins, Nucl. Phys. B 677 (2004) 289 [hep-th/0307203] [SPIRES]. 